\title{
Modeling and simulation of an array of Dielectric Elestomeric Actuator Membranes
}

\author{
Sipontina Croce1, Julian Neu${ }^{1}$, Jonas Hubertus'2, Stefan Seelecke ${ }^{1,3}$, Günter Schultes ${ }^{2}$, and \\ Gianluca Rizzello' \\ ${ }^{1}$ Department of Systems Engineering, Department of Materials Science and Engineering, Saarland University, \\ 66123 Sa arbruecken, Germany, sipontina.croce@imsl.uni-saarland.de \\ 2 University of Applied Sciences of Saarland, Department of Sensors and Thin Films, Goebenstraße 40, \\ Saarbrücken \\ ${ }^{3}$ Center for Mechatronics and Automation Technologies (ZeMA) gGmbH, Sa arbrücken, Germany
}

\begin{abstract}
Recent advances in miniaturized actuators and sensors have enabled the development of cooperative systems, in which a complex global task is achieved through the joint collaboration of several micro-units. Achieving system miniaturization while maintaining the desired actuation/sensor and cooperative functionality, however, is generally quite challenging from the practical point of view. Smart material transducers based on dielectric elastomer (DE) membranes represent a technology with great potential for the design of high-performance microactuator systems. By designing a miniaturized array of DE taxels, their simultaneous actuation and sensing capabilities can be used to develop large deformation, energy-efficient, multi-functional, and cooperative systems. In addition, the high flexibility of DE material makes the developed system highly suitable for new application fields, such as wearables and soft robotics. To properly design, optimize, and control cooperative DE systems, accurate mathematical models need to be developed first. In this paper, we present a novel physics-based model for an array of three DE actuator taxels. Such model represents the first step towards the development, optimization, and control of a complex cooperative matrix actuator. Through the proposed model, it is possible to describe the coupling existing between the DE elements, and predict how such coupling effects influences the complete system performance. After presenting the model, the effect of geometrical parameters on the spatial coupling response is studied by means of numerical simulations.
\end{abstract}

Keywords: dielectric elastomer, DE, modeling, simulation, array, cooperation.

\section{Introduction}

A Dielectric elastomer (DE) transducer consists of an elastomer film sandwiched between two compliant electrodes. DEs can be effectively used as actuators, because they transform electrical energy into motion. By applying a high voltage to the electrodes, a Maxwell stress is generated into the membrane, which results in a thickness reduction and a in subsequent area expansion [1]. Furthermore, due to the correlation existing betw een deformation and electrical capacitance, DEs can also function as sensors. In recent years, DEs have gained significant popularity thanks to their numerous advantages, such as high deformation (on the order of 100\%), high flexibility, lightw eight, energy efficiency, low cost, and self-sensing (i.e., they can work as actuators and as sensors at the same time) [1]. Nowadays, DEs appear as a very promising technology well suited for both for industrial applications (e.g., industrial valves [2,3] or pumps [4,5]) as well as less conventional ones (e.g., loudspeakers [6,7], soft robots [8,9,10], braille displays [11,12], medical robots [13], and artificial muscles [14], to mention few examples).

Despite a number of DE applications has been successfully developed and validated, up to now they are mostly operated at the macroscale level. Only few examples of micro-scale DE devices have 
been currently presented, e.g., [15]. This general lack of micro-scale DE systems is mainly due to many practical issues encountered during the miniaturization process. In fact, scaling a DE device while maintaining the desired actuation/sensing functionality and flexibility presents a number of practical and technological challenges. It is remarked how, up to date, many of those issues are still open. Once DE microtechology is properly investigated and understood, it will allow the design of a new generation of micro-robots, micro-optical systems, and wearable devices, possibly capable of intelligent and cooperative features. To effectively understand and optimise such kind of cooperative DE microactuators, accurate models and simulation tools play a key role. Due to the highly non-linear behavior of the material, how ever, modelling of complex DE strcutures is generally highly involved. In addition, the geometric nonlinearities, the electromechanical coupling, and the neighbour effects that exist between adjacent elements in an array actuator further complicate the overall analytical description. We remark, how ever, that the state-of-the-art literature severely lacks of systematically investigations on modeling and performance scalability for cooperative and small-scale DE systems.

As a first step towards this field, in this paper we present the preliminary development of a physics-based model for a cooperative DE structure. The system under investigation consists of three DE actuator (DEA) elements arranged in an array configuration. By properly controlling the different membranes, cooperative actuation/sensing features can be effectively implemented. The development of a model which can be used for system analysis, simulation, and control represents a fundamental step towards this goal. The proposed physics-based model takes into account the kinematic nonlinearities due to geometry and boundary conditions, together with the electromechanical coupling existing between the DEA taxels. These interactions depend on various design parameters, such as the size of the individual membranes, their relative spacing, as well as the mechanical pretensioning mechanism. After developing the constitutive equations, a numerical study is performed in order to understand the relationship between several design parameters (e.g., geometry, pre-stretch, mechanical bias) and the behaviour of the considered array, focusing on the quasi-static actuation performance.

The remainder of this paper is organized as follows. In Section 2, a simulation model for an array of three actuators composed by DE membranes (DEA) is developed. Then, in order to understand the relationship between the geometry and the behaviour of the overall system, a parameter study is conducted in Section 3. Finally, concluding remarks are discussed in Section 4.

\section{Methods}

This section presents the model of an array of three DEA elements. Figure 1 reports a top-view sketch of such a system. A cross-sectional depiction of the array is illustrated in Figure 2, based on the section determined by the dashed line in Figure 1.

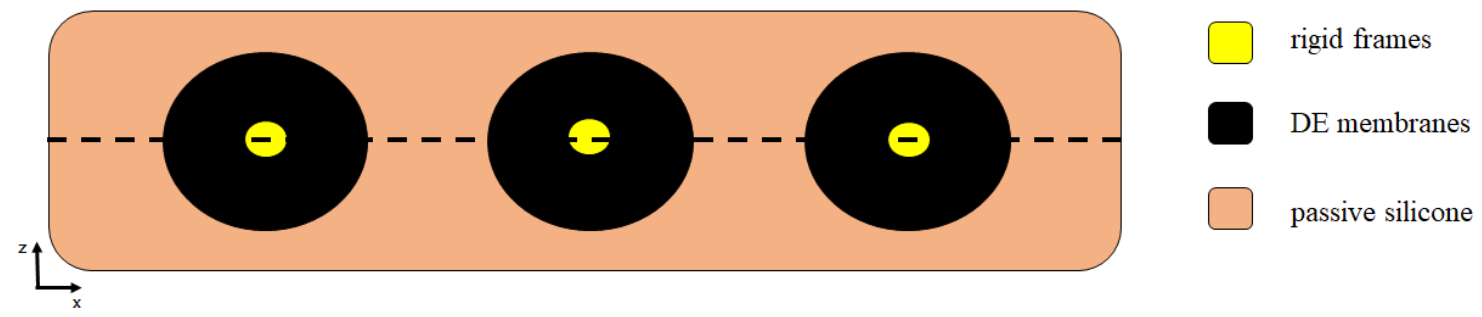

Figure 1. Top view of the DEAs array. The dashed line represents the transversal plane on which the model is based. 


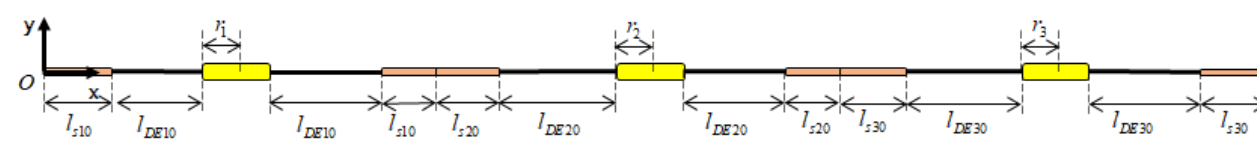

(a)
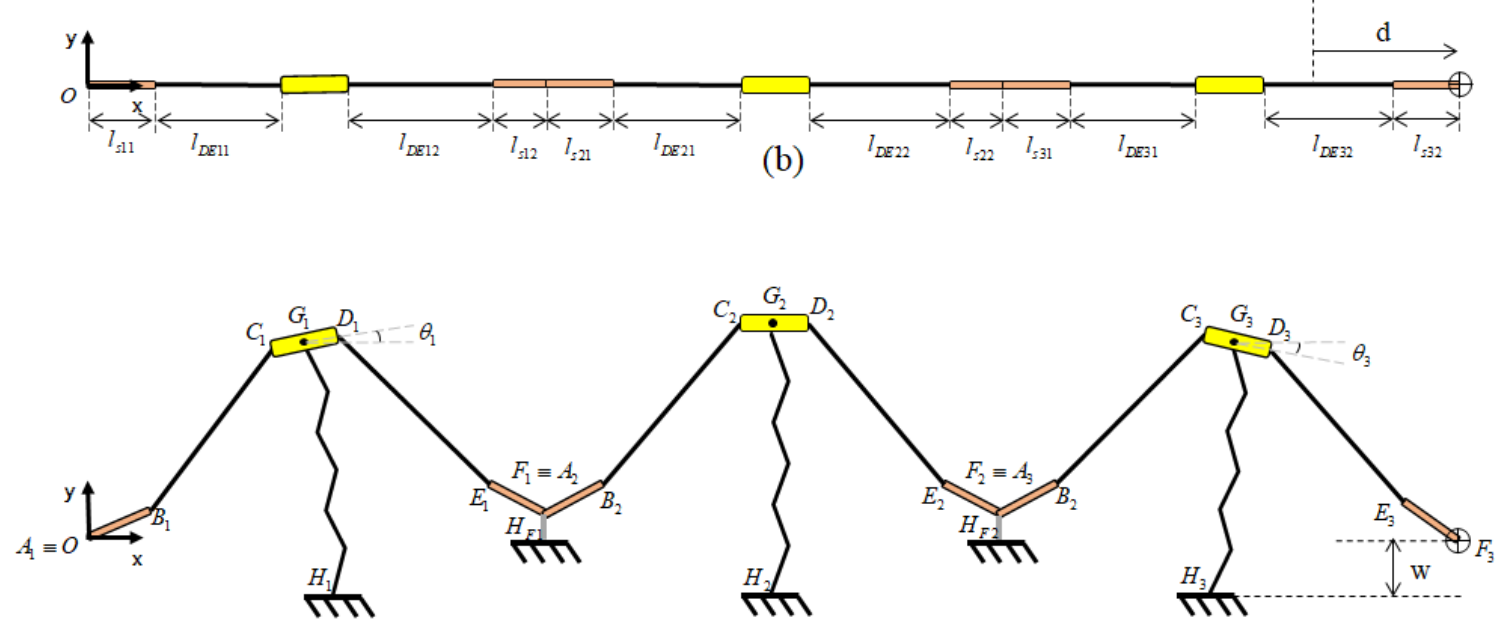

(c)

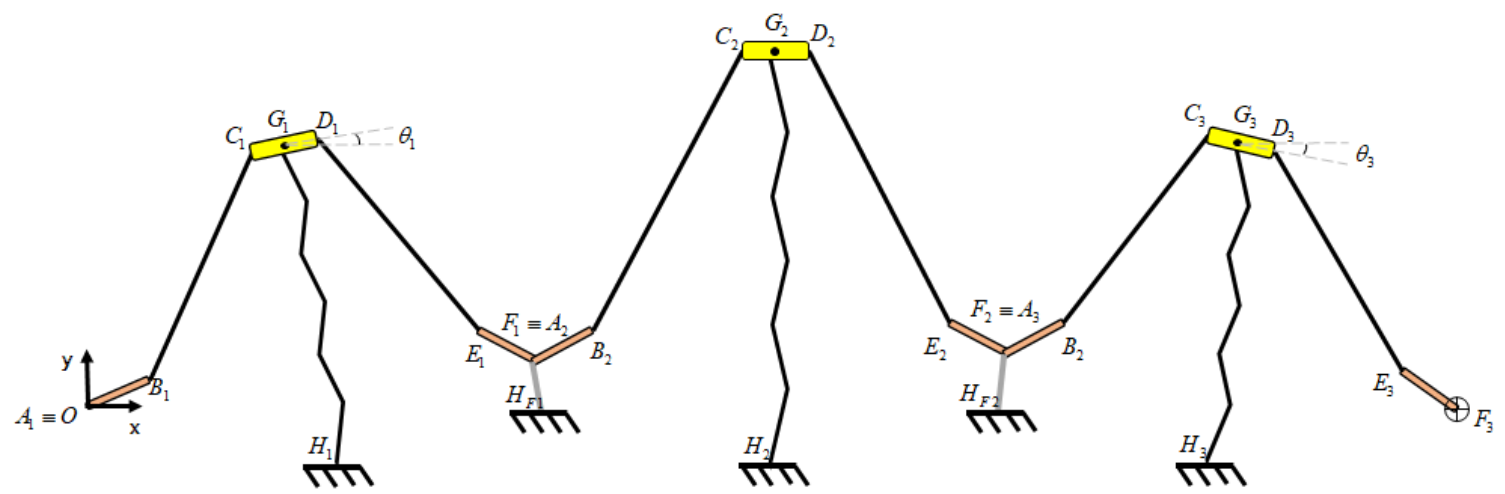

(d)

Figure 2. 2-D cross-sectional view of DEAs array: (a) Undeformed configuration; (b) configuration after horizontal pre-stretch; (a) configuration after adding biasing systems; (d) configuration after electrical activation of the second DEA.

The system consists of three circular DE membranes (depicted in black) printed on a passive silicone membrane (depicted in brown). The left- and right-hand sides of the passive silicone membranes are rigidly clamped to a fixed frame (not shown in Figure 1). Each DEA is preloaded outof-plane with a biasing mechanism, i.e., a pre-compressed linear spring. The deformation of the actuators is guided via a circular rigid frame located at the center part of each DE membrane(depicted in yellow ), which is also used to ensure a connection with the biasing elements. Finally, in the regions of silicone which connect tw o adjacent membranes, additional elastic elements are insterted (depicted in grey). Such elements are used to represent possible compliant connections between the silicone membrane and a rigid frame. They will be particularly useful to understand how the stifffness of those connecting element influences the interaction among neighbor actuators.

To reflect the overall system behavior, four different phases are considered in Figure 2:

- The membrane is initially flat (a);

- An in-plane pre-stretch is applied to the flat membrane (b);

- $\quad$ An out-of-plane force is supplied via the biasing springs (c); 
- $\quad$ The pre-stretched and biased DEA is finally actuated (d).

The purpose of phases (b) and (c) is to obtain a radial preload force to which each DE membrane is subject. This force depends on DE geometry (internal radius, external radius, material stiffness), amount of in-plane pre-stretch, and biasing spring parameters (undeformed length and stiffness). The application of such a preloading force is important, indeed, in order to achieve an actuating stroke. After establishing an equilibrium between the forces of the pre-stretched membrane and the biasing system, the DEAs can be actuated by applying a high voltage. As explained above, the activated membrane increases its surface area as a result of the Maxwell stress. This effect can be also interpreted as a voltage-induced membrane softening, since the in-plane force tends to decrease if the deformation is kept constant. A force imbalance is then created between the elastomer and the biasing element, which, in turn, displaces the DEA rigid spacer upwards until a equilibrium configuration is achieved. The resulting actuated configuration is reported in Figure 2 (d).

\subsection{System modeling}

To better understand the relationship between geometry and actuation performance, as well as the mutual interaction among the DEAs, a physics-based model is developed in this section. For simplicity, the model describes a two-dimensional section of the array (according to Figure 2), rather than the full three-dimensional system. In this way, it it possible to capture all the essential actuation features with a good trade-off between complexity and accuracy. The spacers are described as rigid bodies, capable of both translation and rotation on the plane. Each DEA and silicone element is modeled as a lumped linear spring, whose reaction force depends on the amount of stretching. As a further modeling assumption, the electrical DE activation is implemented as an equivalent stiffness reduction in the corresponding spring element.

Figure 2 illustrates the main geometric parameters of the system in geometrical different configurations, i.e., undeformed membrane (a), pre-stretched and unbiased membrane (b), and full biased system. These parameters are listed below:

- $r_{1,2,3}$ : DE inner radius of the membrane

- $\quad l_{s i 0}, \mathrm{i} \in\{1,2,3\}$ : initial length of the silicone membrane $i$;

- $\quad l_{D E i 0}, i \in\{1,2,3\}$ : external radius of the DE membrane $i$;

- $d$ : horizontal pre-stretch carried out during the first phase;

- $O$ : origin of the fixed reference, with they-axis oriented orthogonally to the ground;

- $w$ : distance between the point for biasing system attachment and the origin $O$;

- $G$ i, $i \in\{1,2,3\}$ : coordinates of the center of mass of the rigid spacer $i$, with respect to the origin $\mathrm{O}$;

- $\quad \theta$ : angle of rotation of the rigid frame with respect to its center of mass;

- $l_{D E i j}, \mathrm{i} \in\{1,2,3\}, \mathrm{j} \in\{1,2\}:$ equivalent length of the DE membrane, where $i$ indicates the actuator and $j$ the element.

Other relevant parameters, that do not appear in Figure 2, are given in the following:

- $\quad d_{i 0}, \mathrm{i} \in\{1,2,3\}$ : undeformed length of the byasing system;

- $k_{B i}, \mathrm{i} \in\{1,2,3\}$ : rigidity constant of the biasing systems;

- $k_{F i}, \mathrm{i} \in\{1,2\}$ : rigidity constant of the central element, positioned between the actuators;

First of all, independent models are produced for the DE membranes and the structural elements, based on an energetic approach. Then, the full electro-mechanically coupled model that describes the complete system is generated. Since in this paper we focus on the prediction of the quasi-static performance, only the characterization of the potential energy is relev ant for the model development. To describe the system configuration, the following vector $q$ of generalized variables are chosen: 


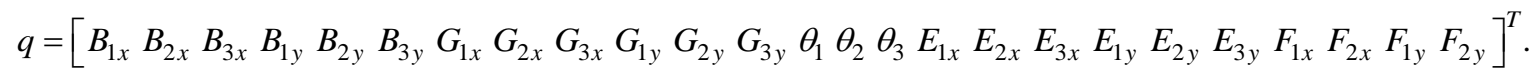

All the variables appearing in (1) are represented in Figure 2(c). On the basis of (1), as well as the constant geometric parameters defined above, we determine the coordinates of the remaining points in Figure 2(c) with respect to the fixed reference $O$, as follows:

$$
\begin{gathered}
A_{1}=(0,0), \\
A_{i}(q)=\left(F_{i x}, F_{i y}\right), i=2,3, \\
C_{i}(q)=\left(G_{i x}-r_{i} \cos \theta_{i}, G_{i y}-r_{i} \sin \theta_{i}\right), i=1,2,3, \\
D_{i}(q)=\left(G_{i x}+r_{i} \cos \theta_{i}, G_{i y}+r_{i} \sin \theta_{i}\right), i=1,2,3, \\
F_{3}=\left(\sum_{i}^{3} 2 l_{S i 0}+\sum_{i}^{3} 2 l_{D E i 0}+\sum_{i}^{3} 2 r_{i}+d, 0\right) .
\end{gathered}
$$

The force of each flexible element (i.e., DEA and passive silicone) can be expressed as a function of the corresponding length. In compact form, we can express the vector of DE lengths $l_{D E}$ and silicone lengths $l_{s}$ as a function of $q$, as follows:

$$
\begin{gathered}
l_{D E}(q)=\left[\begin{array}{l}
l_{D E 11}(q) \\
l_{D E 12}(q) \\
l_{D E 21}(q) \\
l_{D E 22}(q) \\
l_{D E 31}(q) \\
l_{D E 32}(q)
\end{array}\right]=\left[\begin{array}{l}
\left\|C_{1}(q)-B_{1}(q)\right\| \\
\left\|E_{1}(q)-D_{1}(q)\right\| \\
\left\|C_{2}(q)-B_{2}(q)\right\| \\
\left\|E_{2}(q)-D_{2}(q)\right\| \\
\left\|C_{3}(q)-B_{3}(q)\right\| \\
\left\|E_{3}(q)-D_{3}(q)\right\|
\end{array}\right], \\
l_{S}(q)=\left[\begin{array}{l}
l_{S 11}(q) \\
l_{S 12}(q) \\
l_{S 21}(q) \\
l_{S 22}(q) \\
l_{S 31}(q) \\
l_{S 32}(q)
\end{array}\right]=\left[\begin{array}{c}
\left\|A_{1}-B_{1}(q)\right\| \\
\left\|E_{1}(q)-F_{1}(q)\right\| \\
\left\|A_{2}(q)-B_{2}(q)\right\| \\
\left\|E_{2}(q)-F_{2}(q)\right\| \\
\left\|A_{3}(q)-B_{3}(q)\right\| \\
\left\|E_{3}(q)-F_{3}\right\|
\end{array}\right],
\end{gathered}
$$

Next, we need to characterize the system potential energy $\mathcal{V}$ as a function of $q$. This quantity can be decomposed in the sum of several contributions, representing the potential energy of the rigid frames $\mathcal{V}_{R F}$, the DE membranes $\mathcal{V}_{D E}$, the silicone membranes $\mathcal{V}_{S}$, the biasing springs $\mathcal{V}_{B}$, and the frame stiffness elements $\mathcal{V}_{F}$, as follows

$$
\mathcal{V}(q)=\mathcal{V}_{R F}(q)+\mathcal{V}_{D E}(q)+\mathcal{V}_{S}(q)+\mathcal{V}_{B}(q)+\mathcal{V}_{F}(q)
$$

The first contribution in (9) solely consists of the gravitational potential energy of each frame, expressed as follows

$$
\mathcal{V}_{R F}(q)=\sum_{i=1}^{3} m_{R F i} g G_{i y},
$$

where mRFi represents the mass of rigid frame $i$, and $g$ is the gravitational acceleration. The DE potential energy can be simply modeled as folows 


$$
\mathcal{V}_{D E}(q)=\sum_{i=1}^{3} \sum_{j=1}^{2} \frac{1}{2} k_{D E i j}\left(l_{D E i j}(q)-l_{D E i 0}\right)^{2}+\sum_{i=1}^{3} m_{D E i} g \frac{1}{2}\left(C_{i y}+B_{i y}\right)+\sum_{i=1}^{3} m_{D E i} g \frac{1}{2}\left(D_{i y}+E_{i y}\right),
$$

where the first term describes the elastic potential energy, in which $k_{S i j}$ and $l_{D E i}$ represent the stiffness and the undeformed length of the corresponding DE membrane (cf. Figure 2(a)), while the second and third terms describe the potential gravitational energy associated to the DEmass MDEi. In a similar way, we can also represent the potential energy of the silicone in the following way

$$
\mathcal{V}_{S}(q)=\sum_{i=1}^{3} \sum_{j=1}^{2} \frac{1}{2} k_{S i j}\left(l_{S i j}(q)-l_{S i 0}\right)^{2}+\sum_{i=1}^{3} m_{S i} g \frac{1}{2}\left(B_{i y}+A_{i y}\right)+\sum_{i=1}^{3} m_{S i} g \frac{1}{2}\left(E_{i y}+F_{i y}\right),
$$

where $k_{s i j}$ and $l_{s i 0}$ represent the corresponding silicone stiffness and undeformed lengths, while $m s i$ and $l_{\text {sio }}$ represent the corresponding length in the undeformed state (cf. Figure 2(a)). Finally, the potential energy of the biasing springs and the frame connectors is modeled as follows

$$
\begin{gathered}
\mathcal{V}_{B}(q)=\sum_{i=1}^{3} \frac{1}{2} k_{B i}\left(\left\|G_{i}-H_{i}\right\|-d_{i 0}\right)^{2}, \\
\mathcal{V}_{F}(q)=\sum_{i=1}^{2} \frac{1}{2} k_{F i}\left\|F_{i}-H_{F i}\right\|^{2},
\end{gathered}
$$

where $k_{B i}$ and $k_{F i}$ represent the corresponding stiffness of biasing system and central elastic elements, while $d_{i 0}$ represent the corresponding linear spring length in the undeformed state. Additional quantities $G_{i}, H_{i}$, and $H_{F i}$ represent coordinates of the center of mass of the rigid frame $i$, the clamping point of the biasing system, and of the central elastic element (cf. Figure 2(a)).

Once the total potential energy is available, the constitutive equations of the system can be computed by solving the following equilibrium condition (the result follows immediately from Lagrangian mechanics)

$$
\frac{\partial \mathcal{V}(q)}{\partial q}=0
$$

Note that (15) consists of a system of nonlinear algebraic equations. By solving (15) for $q$ via numerical methods, the corresponding system configuration can be computed. Note how the activation of DEA $i$ is modeled as a reduction of $k_{D E i j}$. In this way, we can solve equation (15) for different values of $k_{D E} i j$, and compute the corresponding equilibrium configuration in both unactuated and actuated states.

\section{Results and discussion}

The simulation model, characterised in the previous section, is used to identify the relationship existing between the variation of the geometric parameters and the final configuration of the whole system considered. The simulations are conducted by considering the following nominal parameters values, chosen in order to reflect the typical behavior of DE systems:

- $d=0.01 \mathrm{~m}$;

- $w=-0.01 \mathrm{~m}$;

- $\quad r_{i}, \mathrm{i} \in\{1,2,3\}=0.0025 \mathrm{~m}$;

- $\quad l_{D E i}, \mathrm{i} \in\{1,2,3\}=0.01 \mathrm{~m}$;

- $\quad l$ sio, $\mathrm{i} \in\{1,2,3\}=0.01 \mathrm{~m}$;

- $m D E i, i \in\{1,2,3\}=0.0118 \mathrm{~g}$;

- $m s i, \mathrm{i} \in\{1,2,3\}=0.028 \mathrm{~g}$;

- $m_{R F i}, \mathrm{i} \in\{1,2,3\}=10 \mathrm{~g}$; 
- $k_{D E i}, \mathrm{i} \in\{1,2,3\}=19.6 \mathrm{kN} / \mathrm{m}$;

- $k_{s i}, \mathrm{i} \in\{1,2,3\}=19.6 \mathrm{kN} / \mathrm{m}$;

- $k_{B i}, \mathrm{i} \in\{1,2,3\}=10 \mathrm{MN} / \mathrm{m}$;

- $k_{F F}, \mathrm{i} \in\{1,2\}=0 \mathrm{~N} / \mathrm{m}$;

- $d_{i 0}, \mathrm{i} \in\{1,2,3\}=0.02 \mathrm{~m}$.

The DEA activation is simulated by reducing $k_{D E i}$ by a factor of $20 \%$.

Simulations are conducted by keeping all parameters constant at nominal value and changing only one of them at each iteration, setting different values listed below :

- $\quad \alpha=\left[10^{-6}, \ldots, 1^{6}\right]$ : ratio between the stiffness of the elastic element placed in the centre between two DE membranes $\left(k_{F}\right)$ and the silicone stiffness $(k s)$;

- $k_{B i}=\left[10^{3}, \ldots, 10^{8}\right]$ : stiffness of the biasing system;

- $\mu=[20 \%, \ldots, 80 \%]$ : percentage variation of the DE length $\left(l_{D E i 0}\right)$ relative to the sum of the $\mathrm{DE}$ and silicone lengths $\left(3 l_{D E i 0}+3 l_{s i i}\right)$ (this value will be kept as constant throughout the study).

These values are chosen in such a way to establish a useful sub-range, which show show the steadystate values of the rigid frames vertical positions (i.e., $G_{1 y}, G_{2 y}, G_{3} y$ ) undergo variations when the above listed parameters vary. Thus, it will be possible to understand which geometric value needs to set in input to obtain a desired output configuration.

The results are shown in Figure 3- 5, where the first and second lines show the output obtained by activating the second and third DEA, respectively. The steady state value os computed as the the corresponding equilibrium value of $G_{i y}$, while the stroke is calculated as the absolute value of the corresponding variable before and after the actuation. In Figure 3, we can see that for high $k_{F i}$ values the stroke of non-actuated membranes is zero, while a coupling effect is observed for low $k_{F i}$ values. This indicates that the softer the central elastic element, the greater the influence that the actuation of one DEA has on the other two. Furthermore, it is interesting to note that for low $k_{F}$ values, we alw ays get $G_{2 y}$ (central DEA) values are greater than both $G_{1 y}$ and $G_{3 y}$ (side DEAs). On the other hand, we observe the opposite situation if we only look at the stroke stroke is concerned. This is due to the fact that, when the stiffness of the central element is too low, the second actuator will very close to a singular configuration (i.e., a quasi-flat DEA membrane which prevents an in-plane Maxw ell stress to be transmitted out-of-plane), causing a zero stroke during its actuation and a greater influence on the strokes of the neighbours.

Based on these considerations, we decided to continue the study of the remaining parameters considering by completely the absence of the central element (i.e., $k_{F i}=0$ ). In this way, we aim at emphasizing the mutual dependencies among neighbor actuators. In fact, extending the explanation of the singular configuration also to these studies, it is evident that the effect of the actuation of the second DEA is more strongly affected than the remaining ones (Figure 4). Furthermore, it can be seen that increasing the stiffness of the biasing sy stem leads to larger steady-state values, but at the same time to a zero stroke (thus making this phase not meaningful).

Finally, in Figure 5 it can be seen that as the relative amount of DE increases, the stroke increases as well. This is expected, since the greater the portion of the membrane softened induced by the applied voltage, the greater the expected stroke. 

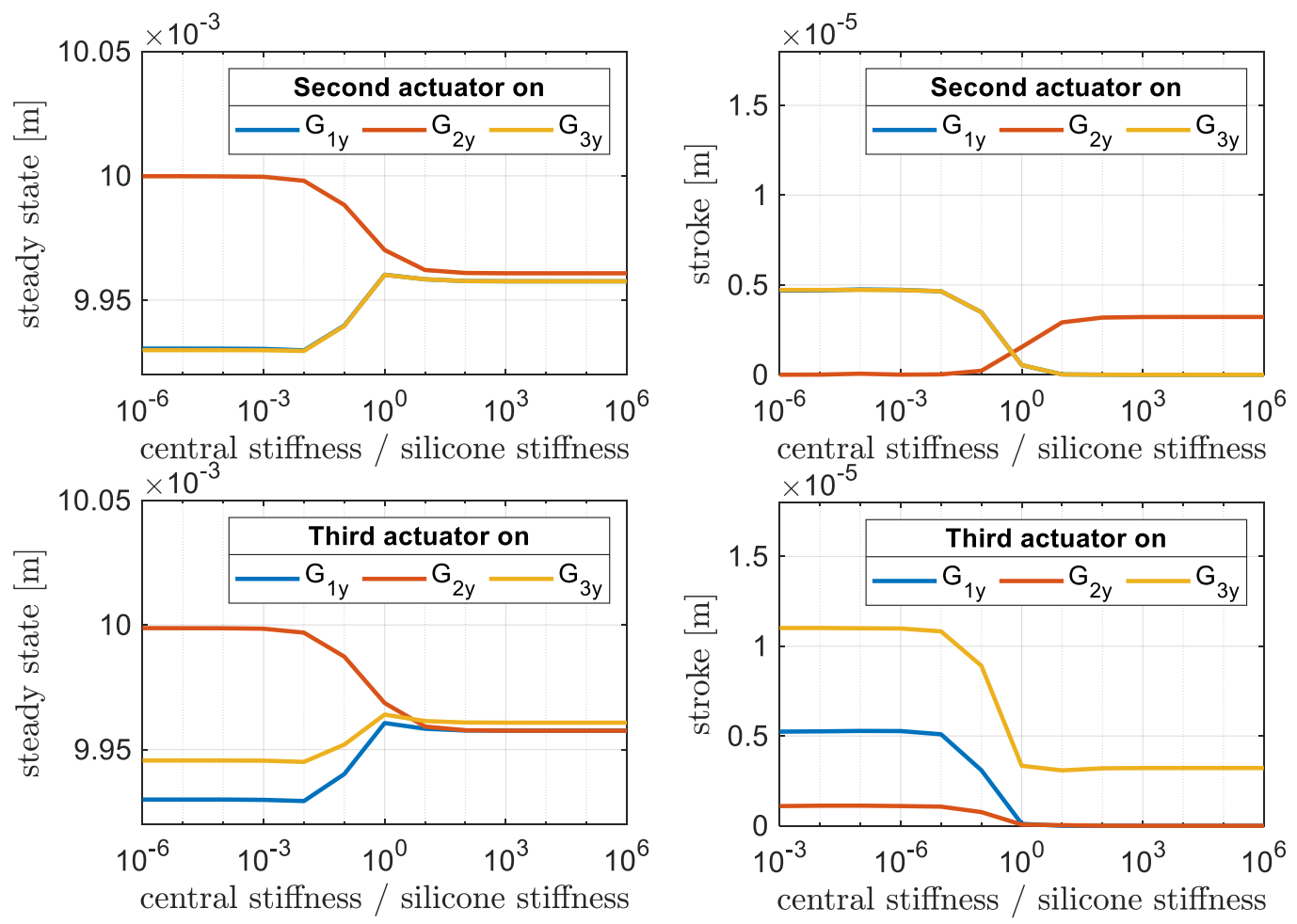

Figure 3. Steady values of $G_{1 y}, G_{2 y}, G_{3 y}$, (left-hand side) and corresponding vertical actuation stroke (right-hand side), a chieved by varying the ratio between the stiffness of the frame element $k_{F i}$ and the stiffne ss of the silicone $k_{s i}$.
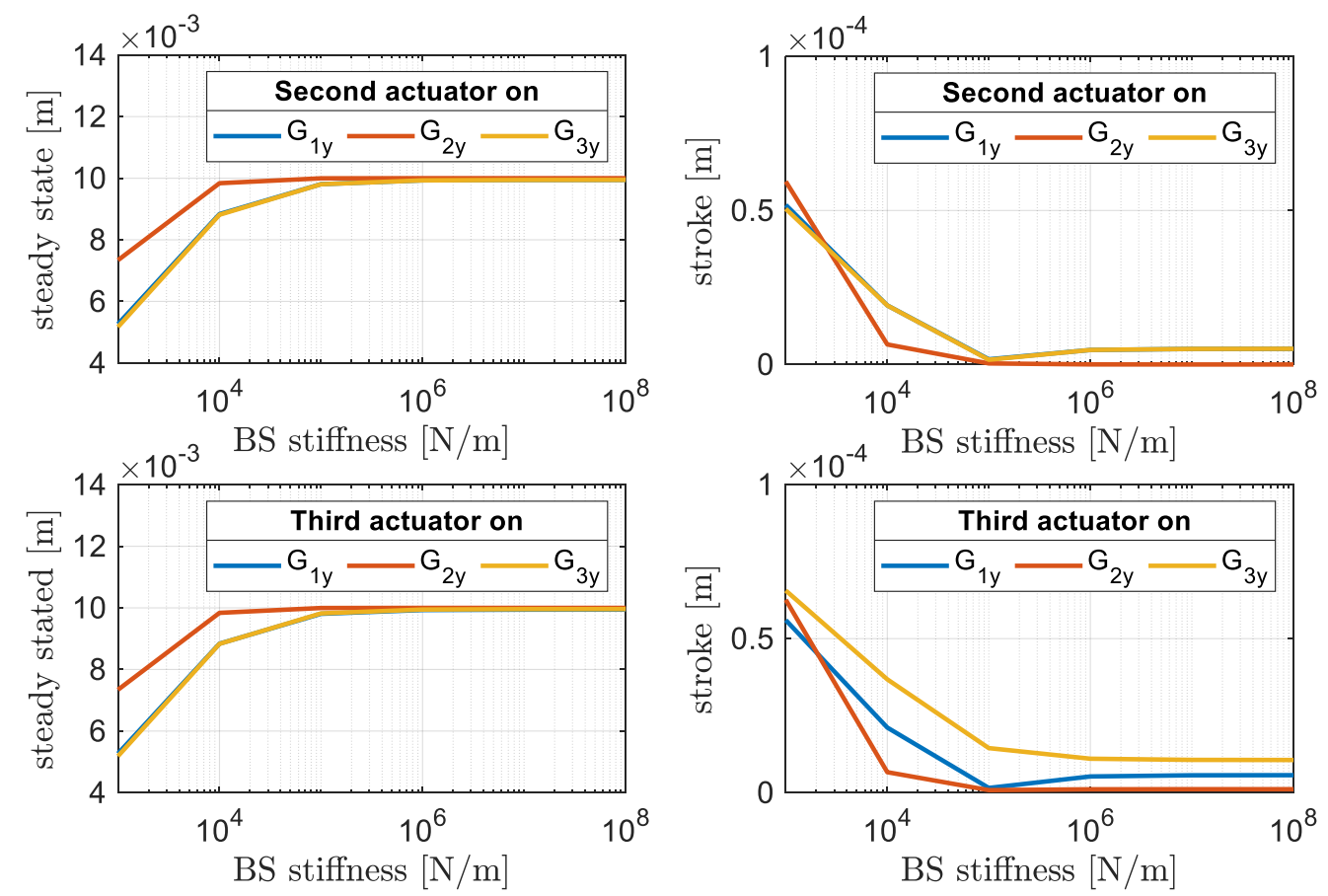

Figure 4. Steady values of $G_{1 y}, G_{2 y}, G_{3 y}$, (left-hand side) and corresponding vertical actuation stroke (right-hand side), a chieved by varying the bia sing system stiffness $k_{B i}$. 

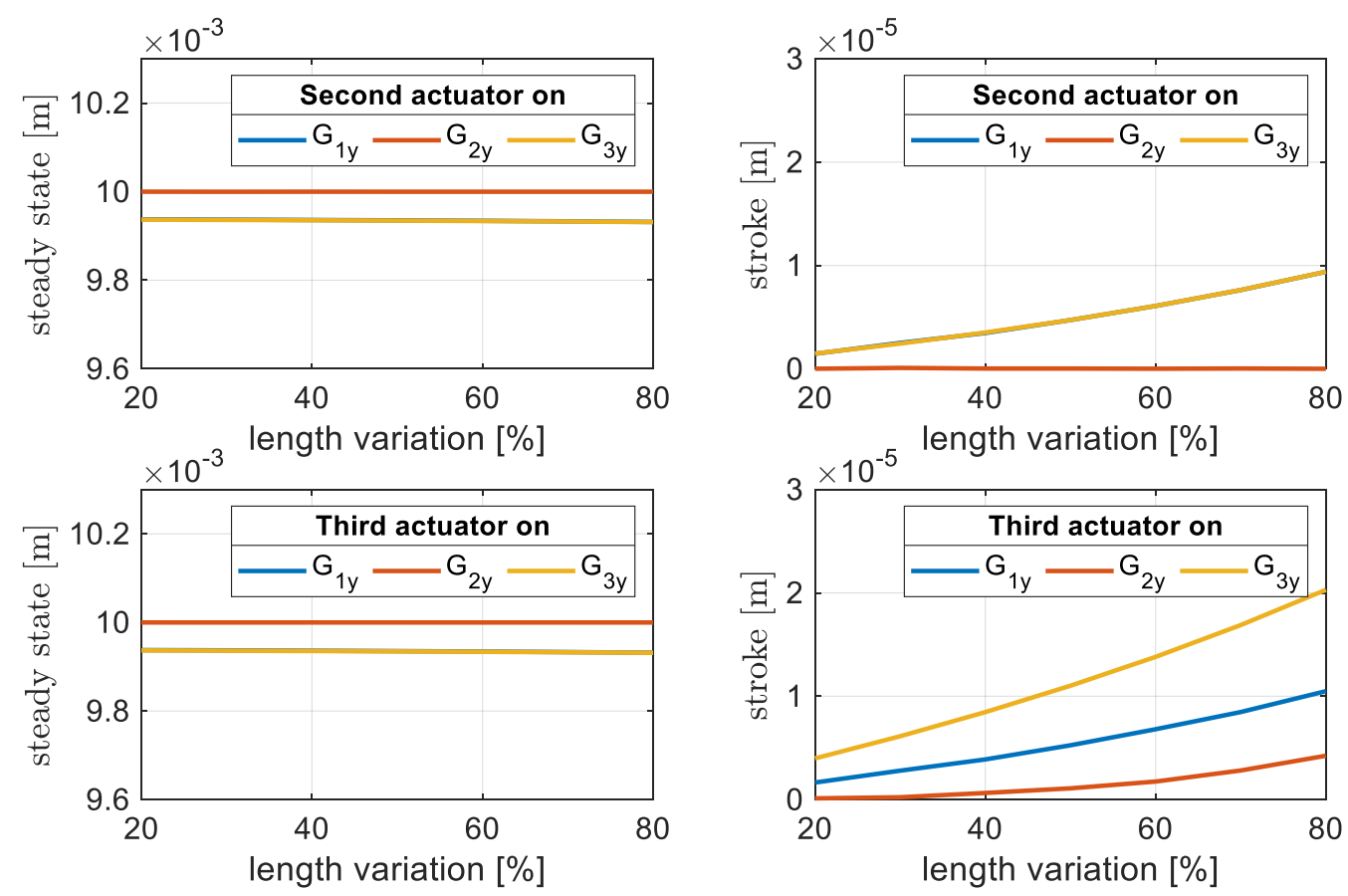

Figure 5. Steady values of $G_{1 y}, G_{2 y}, G_{3 y}$, (left-hand side) and corresponding vertical actuation stroke (right-hand side), a chieved by varying the DE me mbrane external ra dius.

\section{Conclusions}

In this work, we have presented a physics-based model of an array of DEA elements. To deal with thehigh dimensionality of the system (i.e., 25 independent degrees a freedom ), the constitutive equations are obtained via an energetic approach, which allows for a systematic and modular description of the many parts which make up the system. The developed model is useful to accurately understand how the system parameters (i.e., geometry, bias force) affect the mutual electromechanical coupling among the many DEA taxels during actuation.

The provided investigation showed that the softer the connection between DEA taxels and rigid frame, the higher the influences between the actuators. Thanks to this study, it will be possible to identify the optimal geometric combination of values that allows reaching the stroke and a desired configuration, without neglecting the electro-mechanical coupling effects that an actuator has on its neighbors. In addition, it is observed that softer biasing springs and wider DE/passive silicone ratios have an overall positive effect of the actuation stroke. The obtained results can then be used to guide the design and manufacturing of future DEA array systems.

In future research, the developed model will be expanded with dynamic effects describing system inertia and DE viscoelasticity. In addition, more accurate nonlinear models will be adopted for describing both DE and silicone membranes, as well as more advanced types of biasingelements (i.e., bi-stable ones). Experimental validation of the full model will then be conducted. The validated model will then be used the design optimization, scaling, and control of cooperativeDEA systems.

Acknowledgments: The authors gratefully acknowledge the support of the Deutsche Forschungsgemeinschaft (DFG, German Research Foundation) through Priority Program SPP 2206 "Coope rative Multistage Multis table Microactua tor Systems” (Projects: RI3030/2-1, SCHU1609/7-1, SE704/9-1).

Author Contributions: Sipontina Croce performed the simula tions and wrote the paper.

Conflicts of Interest: The authors declare no conflict of inte rest. The founding sponsors had no role in the design of the study; in the collection, analyses, or interpretation of data; in the writing of the manuscript, and in the decision to publish the results.

\section{Abbreviations}

$\mathrm{DE}=$ Dielectric Elastomer; 
$\mathrm{DEA}=$ Dielectric Elastomer Actuators

\section{References}

1. Carpi, F., Rossi, D. D., Kornbluh, R., Pelrine, R. E., and Sommer-Larsen, P., [Dielectric Elastomers as Electromechanical Transducers: Fundamentals, Materials, Devices, Models and Applications of an Emerging Electroactive Polymer Technology], Else vier (Sept. 2011).

2. Hill M, Rizzello G and Seelecke S 2017 De velopment and experimental characterization of a pne umatic valve actuated by a dielectric ela stomer me mbrane Smart Ma ter. Struct. 26085023.

3. Giousouf M and Kovacs G 2013 Dielectric elastomer actuators used for pne uma tic valve technology Smart Mater. Struct. 22104010.

4. Ghazali F A M, Mah C K, AbuZaiter A, Chee P S and Ali M S M 2017 Soft dielectric elastomer actuator micropump Sensors Actuators A Phys. 263 276-284.

5. Loverich J J, Kanno I and Kotera H 2006 Concepts for a new class of all-polymer micropumps Lab Chip 6 1147-1154.

6. Heydt R, Kornbluh R, Eckerle J and Pelrine R 2006 Sound radiation properties of dielectric elastomer electroactive polymer louds peakers Smart Structures and Ma terials 2006: Electroactive Polymer Actuators and Devices (EAPAD) 61681M.

7. Heydt R, Pelrine R, Joseph J, Eckerle J and Kornbluh R 2000 Acoustical performance of an electrostrictive polymer film loudspeakerJ. Acoust. Soc. Am. 107 833-839.

8. Conn A T, Hinitt A D and Wang P 2014 Soft segmented inchworm robot with dielectric elastomer muscles Electroactive Polymer Actua tors and Devices (EAPAD) 90562L

9. Shintake J, Rosset S, Schubert B, Floreano D and Shea H R 2016 Versatile Soft Grippers with Intrinsic Electroadhesion Based on Multifunctional Polymer Actuators Adv. Mater. 28 231-238.

10. Prechtl Johannes, Kunze Julian, Nalbach Sophie, Seelecke Stefan, Rizzello Gianluca, 2020/04/22, Soft robotic module actuated by silicone-based rolled dielectric elastomer actuators: modeling and simulation, 10.1117/12.2557736.

11. Maffli L, RossetS and Shea HR 2013 Mm-size bistable zipping dielectric elastomer actuators for integrated microfluidics Electroactive Polymer Actuators and Devices (EAPAD) 86872M.

12. Chakraborti P, Toprakci H A K, Yang P, Di Spigna N, Franzon P and Ghosh T 2012 A compact dielectric elastomer tubular a ctua tor for refreshable Braille dis plays Sensors Actua tors A Phys. 179 151-157.

13. Carpi, F, Frediani, G, De Rossi, D (2009), Electroactive elastomeric haptic displays of organ motility and tissue compliance for medical training and surgical force feedback, IEEE Transaction Biomedical Engineering 56(9): 2327-2330.

14. Kovacs G2006 Arm Wrestling Robot Driven by Dielectric Elastomer Actuators The First IEEE/RAS-EMBS Int. Conf. Biomed. Robot. Biome chatronics 260-265.

15. Aksoy Bekir, Shea Herbert, 2020/05/20, Reconfigurable and Latchable Shape-Morphing Dielectric Elastomers Based on LocalStiffness Modulation, 10.1002/adfm.202001597. 